\title{
Impact of an emergency department resident strike during the coronavirus disease 2019 (COVID-19) pandemic in Daegu, South Korea: a retrospective cross- sectional study
}

\author{
Yo Han Cho ${ }^{1}$, Jae Wan Cho ${ }^{1}$, Hyun Wook Ryoo ${ }^{1}$, Sungbae Moon ${ }^{1}$, Jung Ho Kim ${ }^{2}$, Sang-Hun Lee ${ }^{3}$, \\ Tae Chang Jang ${ }^{4}$, Dong Eun Lee ${ }^{5}$ \\ ${ }^{1}$ Department of Emergency Medicine, Kyungpook National University Hospital, School of Medicine, Kyungpook National University, Daegu, \\ Korea \\ ${ }^{2}$ Department of Emergency Medicine, Yeungnam University College of Medicine, Daegu, Korea \\ ${ }^{3}$ Department of Emergency Medicine, Keimyung University Dongsan Medical Center, Daegu, Korea \\ ${ }^{4}$ Department of Emergency Medicine, Daegu Catholic University School of Medicine, Daegu, Korea \\ ${ }^{5}$ Department of Emergency Medicine, Kyungpook National University Chilgok Hospital, Daegu, Korea
}

\begin{abstract}
Background: To prepare for future work stoppages in the medical industry, this study aimed to identify the effects of healthcare worker strikes on the mortality rate of patients visiting the emergency department (ED) at six training hospitals in Daegu, South Korea. Methods: We used a retrospective, cross-sectional, multicenter design to analyze the medical records of patients who visited six training hospitals in Daegu (August 21-September 8, 2020). For comparison, control period 1 was set as the same period in the previous year (August 21-September 8, 2019) and control period 2 was set as July 1-19, 2020. Patient characteristics including age, sex, and time of ED visit were investigated along with mode of arrival, length of ED stay, and in-hospital mortality. The experimental and control groups were compared using $t$-tests, and Mann-Whitney U-test, chi-square test, and Fisher exact tests, as appropriate. Univariate logistic regression was performed to identify significant factors, followed by multivariate logistic regression analysis.

Results: During the study period, 31,357 patients visited the ED, of which 7,749 belonged to the experimental group. Control periods 1 and 2 included 13,100 and 10,243 patients, respectively. No significant in-hospital mortality differences were found between strike periods; however, the results showed statistically significant differences in the length of ED stay.

Conclusion: The ED resident strike did not influence the mortality rate of patients who visited the EDs of six training hospitals in Daegu. Furthermore, the number of patients admitted and the length of ED stay decreased during the strike period.
\end{abstract}

Keywords: COVID-19; Emergency medical services; Employee strikes; Hospital mortality; Hospitals; Physicians

Received: May 12, 2021 • Revised: July 5, 2021 • Accepted: July 9, 2021

Corresponding author: Jae Wan Cho, MD

Department of Emergency Medicine, Kyungpook National University Hospital, School of Medicine, Kyungpook National University, 130 Dongdeok-ro, Junggu, Daegu 41944, Korea

Tel: +82-53-200-6400• Fax: +82-53-428-2820•E-mail: jaewanem@knuh.kr 


\section{Introduction}

In August 2020, residents across the country went on strike to protest against the government's healthcare policies. Although a labor strike is a worker's right, healthcare worker strikes concern public welfare and are, therefore, a controversial social issue. A healthcare worker strike can cause public concern, as it has the potential to disrupt normal healthcare delivery, halt disease testing and treatment, and lead to increased overall mortality. However, previous studies have reported that mortality is not markedly altered during a healthcare worker strike. In a review by Cunningham et al. [1], four studies reported reduced mortality, and three studies reported no difference in mortality during a physicians' strike. A study conducted in Kilifi, Kenya, using data registered in the Kilifi Health and Demographic Surveillance system, revealed no considerable change in mortality during the six physician and nurse strikes that took place between 2010 and 2016. [2]. One Korean study from 2000 reported that emergency department (ED) use and length of ED stay decreased during an ED healthcare providers' strike [3]. The year 2020 was a period of dramatic changes for patients and healthcare providers alike because of the coronavirus disease 2019 (COVID-19) pandemic. Hartnett et al. [4] reported that the number of patients visiting the ED decreased, and the reason for ED visits showed marked changes during the COVID-19 pandemic.

Furthermore, another study reported that ED utilization declined out of fear of contracting COVID-19 at the ED and that the number of out-of-hospital cardiac arrests increased [5]. Amidst the pandemic, medical residents in South Korea went on strike for 19 days in opposition to the government's healthcare policies.

Previous studies have generally analyzed the relationship between strikes and patient mortality rates. In the present study, we investigated the effects of the resident strike during the COVID-19 pandemic on patient mortality, as well as the general characteristics of patients visiting the EDs of six teaching hospitals in Daegu, South Korea, ultimately presenting data to help respond to potential healthcare worker strikes in the future.

\section{Methods}

Ethical statements: This study was approved by the Institutional Review Board (IRB) of the Kyungpook National University Hospital (IRB No. 2020-12-024) and was conducted in accordance with the 1964 Declaration of Helsinki and its later amendments. The requirement for informed consent was waived by the IRB owing to the retrospective nature of the study.

\section{Study design, setting, population, and variables}

This multicenter cross-sectional study retrospectively analyzed the medical records of six teaching hospitals in Daegu Metropolitan City from August 21 to September 8, 2020. Control period 1 (August 21 -September 8,2019 ) was set as the same period in the preceding year to reduce the seasonal influence. Control period 2 (July 1-19, 2020) was set to the same period to consider the impact of COVID-19. The start of control period 2 (July 2020) was set one month before the initiation of the strike (August 2020) for two reasons. First, in the early months of 2020, Daegu experienced a surge in COVID-19 cases. In those months, the effects of COVID-19 on patients' characteristics may be greater than the effects of the strike. The other reason was to choose a month with as minimal seasonal factors as possible. During control period 2, there were fewer than 100 COVID-19 cases per day.

In 2019, 252,608 patients visited the EDs of the six target hospitals. Prior to the strike, 83 physicians ( 46 specialists and 37 residents) worked in the ED. The resident strike participation rate was $100 \%$, and during the strike, all residents in the ED were replaced by specialists. The target patient population included patients presenting to the EDs of six teaching hospitals during the strike and those presenting during the control periods. Patients who did not present to an ED to receive medical care (e.g., those who visited to retrieve their medical records, to obtain medication, or to cancel appointments) and those who were dead on arrival were excluded; patients who received cardiopulmonary resuscitation (CPR) on arrival were also excluded, as this can affect the primary outcome (in-hospital mortality). Patient age, sex, and ED visit time were examined. Factors related to ED visits, including mode of arrival to the ED, time of arrival, route of visit, triage, length of ED stay, disposition, and in-hospital death, were analyzed to determine their effect on the primary and secondary outcomes. The primary outcome was the in-hospital mortality rate, which was defined as death in the ED and death in the hospital following hospitalization. The secondary outcome was the length of ED stay, to examine the impact of the strike on the patient population.

\section{Statistical analyses}

Statistical analyses were performed using IBM SPSS version 25.0 (IBM Corp., Armonk, NY, USA). Categorical variables are presented as frequencies and percentages. Continuous variables are presented as median values with interquartile ranges. When comparing the patient populations between the strike and control periods, normally distributed continuous data were analyzed by $t$-tests, and non-normally distributed continuous data were analyzed using the Mann-Whitney $U$-test. Categorical data were analyzed using the chi-square test and Fisher exact test. Multivariate logistic re- 
gression analysis was used to assess the effects of the predictors on the outcome, hospital mortality (by the period, hospital, Korean Triage and Acuity Scale [KTAS], mode of arrival, age, and patients per bed). All variables with a significance level of $P$ less than 0.01 in the univariate analysis were included in the multivariate logistic regression model. Control period 2 was designated as a reference for intuitive understanding in the multivariate logistic regression analysis. The results of the logistic regression analysis are presented as odds ratios (ORs) and $95 \%$ confidence intervals (CIs). Statistical significance was defined as a $p$-value of $\leq 0.05$.

\section{Results}

A total of 31,357 patients were admitted to the ED during the study period. Patients who visited the ED for nontreatment purposes (patients who required CPR on arrival, visited for medical certification, were dead on arrival, and had canceled their appointment) were excluded. The number of patients who visited the ED was 13,100 during control period 1, 10,243 during control period 2, and 7,749 during the strike period (Fig. 1).

\section{Comparison of control period 1 with the strike period}

The median age of the patients was 50 years in control period 1 and 55 years in the strike period. The most common mode of arrival was individual transportation $(n=8,315,63.5 \%$ vs. $n=4,675$, $60.3 \%)$, followed by public ambulance services $(n=2,688,20.5 \%$ vs. $n=1,861,24.0 \%)$, and private ambulance services $(n=2,097$, $16.0 \%$ vs. $n=1,213,15.7 \%$ ), with differences observed between the two periods. The ED route also differed between the two periods for direct visits $(n=10,638,81.2 \%$ vs. $n=6,424,82.9 \%)$ and transfers $(n=2,462,18.8 \%$ vs. $n=1,325,17.1 \%)$. According to KTAS, the number of patients with level 1 was 119 (0.9\%) vs. 98 (1.3\%), with level 2 was 922 (7.0\%) vs. 699 (9.0\%), and level 3

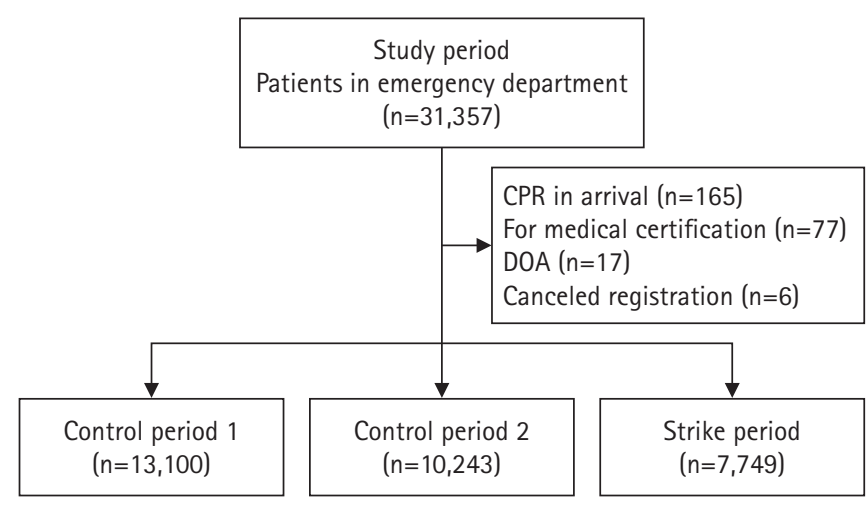

Fig. 1. Flow chart of enrolled patients. CPR, cardiopulmonary resuscitation; DOA, dead on arrival. was 5,932 (45.3\%) vs. 4,096 (52.9\%). Length of stay (LOS) in the ED decreased from 3.2 hours (1.8-6.2 hours) to 3.0 hours (1.66.4 hours). The number of patients per bed significantly decreased from 1.01 to 0.53 . Hospital mortality significantly increased from $258(2.0 \%)$ to 195 patients $(2.5 \%)$ (Table 1$)$.

\section{Comparison of control period 2 with strike period}

The median age of patients was 54 years in control period 2 and 55 years in the strike period, and the difference was not statistically significant. The most common mode of arrival was individual transportation ( $n=6,151,60.1 \%$ vs. $n=4,675,60.3 \%)$, followed by public ambulance services ( $n=2,432,23.7 \%$ vs. $n=1,861,24.0 \%)$, and private ambulance services $(n=1,660,16.2 \%$ vs. $n=1,213$, $15.7 \%)$. The ED route differed between the two periods for direct visits $(\mathrm{n}=8,269,80.7 \%$ vs. $\mathrm{n}=6,424,82.9 \%)$ and transfers $(n=1,974,19.3 \%$ vs. $n=1,325,17.1 \%)$. Time from onset of symptoms to time of visit increased from 5.2 hours (1.3-24.1 hours) to 6.2 hours (1.6-24.8 hours). The number of patients classified into KTAS level 1 was 112 (1.1\%) vs. 98 (1.3\%); level 2 was 849 $(8.3 \%)$ vs. $699(9.0 \%)$, level 3 was 5,412 (52.8\%) vs. 4,096 (52.9\%), and those assigned to levels 4 and 5 combined was 3,870 (37.8\%) vs. 2,856 (36.8\%), showing a reduced proportion of those with mild conditions. Patients' ED LOS decreased from 3.7 hours (2.0-7.6 hours) to 3.0 hours (1.6-6.4 hours). The number of patients per bed decreased from 0.81 patients $(0.64-0.85$ patients) to 0.53 patients ( $0.41-0.55$ patients). Hospital mortality did not differ and was $2.5 \%$ in both the control and strike periods (Table 2).

\section{Multivariate logistic regression of the associated factors for hospital mortality}

Regarding hospital mortality by period, significant differences were observed in the univariate analysis for control period 1, but not in the multivariate analysis (Table 3). The OR for hospital mortality was 98.85 (95\% CI, 61.97-157.65) for patients with KTAS level 1, 18.17 (95\% CI, 11.93-27.66) for those with level 2, and 4.74 (95\% CI, 3.15-7.13) for those with level 3. Regarding the mode of arrival, the OR for hospital mortality was 2.38 (95\% CI, 1.89-2.99) for patients using a public ambulance service and 3.03 (95\% CI, 2.40-3.81) for patients using a private ambulance service as opposed to those using individual transportation, indicating a significant difference. The OR for hospital mortality in terms of age was statistically significant at 1.04 (95\% CI, 1.03-1.04), as was ED LOS at 1.03 (95\% CI, 1.02-1.04).

\section{Discussion}

This study aimed to investigate the impact of a 19-day resident 
Table 1. Demographic and clinical characteristics of the participants in control period 1 and the strike period

\begin{tabular}{|c|c|c|c|}
\hline Characteristic & $\begin{array}{c}\text { Control period } 1 \\
\text { (August 21-September 8, 2019) }\end{array}$ & $\begin{array}{c}\text { Strike period } \\
\text { (August 21-September 8, 2020) }\end{array}$ & $p$-value \\
\hline No. of patients & 13,100 & 7,749 & \\
\hline Age (yr) & $50(22-68)$ & $55(31-71)$ & $<0.01$ \\
\hline Male sex & $6,885(52.6)$ & $4,082(52.7)$ & 0.40 \\
\hline \multicolumn{4}{|l|}{ Vital sign } \\
\hline Mean arterial pressure $(\mathrm{mmHg})$ & $94.7(83.3-106.7)$ & $96.7(83.3-106.7)$ & $<0.01$ \\
\hline Heart rate (beat/min) & $88(76-105)$ & 87 (76-103) & $<0.01$ \\
\hline Respiration rate (breath/min) & $20(20-20)$ & $20(18-20)$ & $<0.01$ \\
\hline Body temperature $\left({ }^{\circ} \mathrm{C}\right)$ & $36.8(36.5-37.3)$ & $36.9(36.5-37.3)$ & $<0.01$ \\
\hline $\mathrm{O}_{2}$ saturation $(\%)$ & 98 (97-99) & 98 (97-99) & 0.36 \\
\hline Hospital & & & $<0.01$ \\
\hline A & $2,652(20.2)$ & $1,442(18.6)$ & \\
\hline B & $2,112(16.1)$ & $1,485(19.2)$ & \\
\hline C & $1,754(13.4)$ & $805(10.4)$ & \\
\hline D & $1,700(13.0)$ & $975(12.6)$ & \\
\hline$E$ & $1,725(13.2)$ & $1,147(14.8)$ & \\
\hline $\mathrm{F}$ & $3,157(24.1)$ & $1,895(24.5)$ & \\
\hline Disease ${ }^{a)}$ & $10,340(78.9)$ & $6,180(79.8)$ & \\
\hline Nondisease & $2,753(21.0)$ & $1,563(20.2)$ & \\
\hline Mode of arrival & & & $<0.01$ \\
\hline Individual transportation & $8,315(63.5)$ & $4,675(60.3)$ & \\
\hline Public ambulance service & $2,688(20.5)$ & $1,861(24.0)$ & \\
\hline Private ambulance service & $2,097(16.0)$ & $1,213(15.7)$ & \\
\hline Time of arrival & & & 0.15 \\
\hline 08:00-15:59 & $5,050(38.5)$ & 3,082 (39.8) & \\
\hline $16: 00-23: 59$ & $5,526(42.2)$ & $3,170(40.9)$ & \\
\hline 00:00-07:59 & 2,524 (19.3) & 1,497 (19.3) & \\
\hline Route of ED arrival & & & $<0.01$ \\
\hline Direct visit & $10,638(81.2)$ & $6,424(82.9)$ & \\
\hline Transfer & 2,462 (18.8) & $1,325(17.1)$ & \\
\hline Onset to visit time (hr) & $6.6(1.4-28.7)$ & $6.2(1.6-24.8)$ & 0.40 \\
\hline KTAS level & & & $<0.01$ \\
\hline 1 & $119(0.9)$ & $98(1.3)$ & \\
\hline 2 & $922(7.0)$ & $699(9.0)$ & \\
\hline 3 & $5,932(45.3)$ & $4,096(52.9)$ & \\
\hline 4,5 & $3,870(29.5)$ & $2,856(36.9)$ & \\
\hline ED LOS (hr) & $3.2(1.8-6.2)$ & $3.0(1.6-6.4)$ & $<0.01$ \\
\hline Patients per bed & $1.01(0.67-1.08)$ & $0.53(0.41-0.55)$ & $<0.01$ \\
\hline Disposition from the $E D^{b)}$ & & & $<0.01$ \\
\hline Discharge & $9,164(70.0)$ & $5,197(67.1)$ & \\
\hline Transfer & $404(3.1)$ & $264(3.4)$ & \\
\hline Admission & $3,450(26.3)$ & $2,221(28.7)$ & \\
\hline Death & $26(0.2)$ & $49(0.6)$ & \\
\hline Hospital mortality & $258(2.0)$ & $195(2.5)$ & 0.01 \\
\hline
\end{tabular}

Values are presented as number only, median (interquartile range), or number (\%).

ED, emergency department; KTAS, Korean Triage and Acuity Scale; LOS, length of stay.

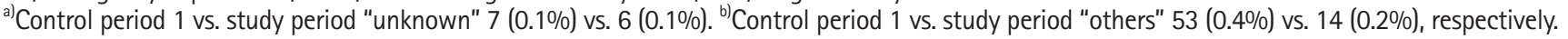


Table 2. Demographic and clinical characteristics of the participants in control period 2 and the strike period

\begin{tabular}{|c|c|c|c|}
\hline Characteristic & $\begin{array}{c}\text { Control period } 2 \\
\text { (July } 1-J u l y ~ 19,2020 \text { ) }\end{array}$ & $\begin{array}{c}\text { Strike period } \\
\text { (August 21-September 8, 2020) }\end{array}$ & $p$-value \\
\hline No. of patients & 10,243 & 7,749 & \\
\hline Age (yr) & $54(29-71)$ & $55(31-71)$ & 0.07 \\
\hline Male sex & $5,311(51.9)$ & $4,082(52.7)$ & 0.27 \\
\hline \multicolumn{4}{|l|}{ Vital sign } \\
\hline Mean arterial pressure $(\mathrm{mmHg})$ & 93.7 (83.3-106.7) & $96.7(83.3-106.7)$ & $<0.01$ \\
\hline Heart rate (beat/min) & $86(76-103)$ & $87(76-103)$ & 0.88 \\
\hline Respiration rate (breath/min) & $20(19-20)$ & $20(18-20)$ & $<0.01$ \\
\hline Body temperature $\left({ }^{\circ} \mathrm{C}\right)$ & $36.8(36.5-37.3)$ & $36.9(36.5-37.3)$ & $<0.01$ \\
\hline $\mathrm{O}_{2}$ saturation $(\%)$ & 98 (97-99) & 98 (97-99) & $<0.01$ \\
\hline Hospital & & & $<0.01$ \\
\hline A & 1,969 (19.2) & 1,442 (18.6) & \\
\hline B & 1,938 (18.9) & $1,485(19.2)$ & \\
\hline C & $1,343(13.1)$ & $805(10.4)$ & \\
\hline D & 1,413 (13.8) & $975(12.6)$ & \\
\hline $\mathrm{E}$ & $1,215(11.9)$ & 1,147 (14.8) & \\
\hline $\mathrm{F}$ & $2,365(23.1)$ & $1,895(24.5)$ & \\
\hline Disease $e^{a)}$ & $8,063(78.7)$ & $6,180(79.8)$ & \\
\hline Nondisease & $2,176(21.2)$ & $1,563(20.2)$ & \\
\hline Mode of arrival & & & $<0.01$ \\
\hline Individual transportation & $6,151(60.1)$ & $4,675(60.3)$ & \\
\hline Public ambulance service & $2,432(23.7)$ & $1,861(24.0)$ & \\
\hline Private ambulance service & $1,660(16.2)$ & $1,213(15.7)$ & \\
\hline Time of arrival & & & 0.84 \\
\hline 08:00-15:59 & 4,038 (39.4) & 3,082 (39.8) & \\
\hline $16: 00-23: 59$ & $4,235(41.3)$ & $3,170(40.9)$ & \\
\hline 00:00-07:59 & 1,970 (19.2) & 1,497 (19.3) & \\
\hline Route of ED arrival & & & $<0.01$ \\
\hline Direct visit & $8,269(80.7)$ & $6,424(82.9)$ & \\
\hline Transfer & $1,974(19.3)$ & $1,325(17.1)$ & \\
\hline Time from onset to visit (hr) & $5.2(1.3-24.1)$ & $6.2(1.6-24.8)$ & $<0.01$ \\
\hline KTAS level & & & $<0.01$ \\
\hline 1 & $112(1.1)$ & $98(1.3)$ & \\
\hline 2 & $849(8.3)$ & $699(9.0)$ & \\
\hline 3 & $5,412(52.8)$ & $4,096(52.9)$ & \\
\hline 4,5 & $3,870(37.8)$ & $2,856(36.8)$ & \\
\hline ED LOS (hr) & $3.7(2.0-7.6)$ & $3.0(1.6-6.4)$ & $<0.01$ \\
\hline Patients per bed & $0.81(0.64-0.85)$ & $0.53(0.41-0.55)$ & $<0.01$ \\
\hline Disposition from the $E D^{b)}$ & & & $<0.01$ \\
\hline Discharge & $6,709(65.5)$ & $5,197(67.1)$ & \\
\hline Transfer & $297(2.9)$ & $264(3.4)$ & \\
\hline Admission & $3,122(30.5)$ & $2,221(28.7)$ & \\
\hline Death & $66(0.6)$ & $49(0.6)$ & \\
\hline Hospital mortality & $253(2.5)$ & $195(2.5)$ & 0.84 \\
\hline
\end{tabular}

Values are presented as number only, median (interquartile range), or number (\%).

ED, emergency department; KTAS, Korean Triage and Acuity Scale; LOS, length of stay.

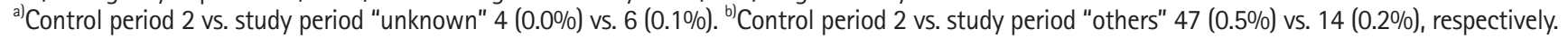


Table 3. Univariate and multivariate logistic regression analyses of associated factors for hospital mortality

\begin{tabular}{|c|c|c|c|c|}
\hline \multirow{2}{*}{ Variable } & \multicolumn{2}{|c|}{ Univariate } & \multicolumn{2}{|c|}{ Multivariate } \\
\hline & OR $(95 \% \mathrm{Cl})$ & $p$-value & $\mathrm{OR}(95 \% \mathrm{Cl})$ & $p$-value \\
\hline \multicolumn{5}{|l|}{ Period } \\
\hline Control period 2 & Reference & & Reference & \\
\hline Control period 1 & $0.79(0.67-0.95)$ & 0.01 & $1.04(0.85-1.28)$ & 0.71 \\
\hline Strike period & $1.02(0.84-1.23)$ & 0.84 & $0.98(0.69-1.38)$ & 0.90 \\
\hline \multicolumn{5}{|l|}{ Hospital } \\
\hline$A$ & Reference & & Reference & \\
\hline B & $0.61(0.48-0.79)$ & $<0.01$ & $0.83(0.63-1.09)$ & 0.18 \\
\hline C & $0.98(0.77-1.25)$ & 0.86 & $0.68(0.47-1.01)$ & 0.05 \\
\hline D & $1.09(0.87-1.38)$ & 0.46 & $1.13(0.77-1.65)$ & 0.53 \\
\hline$E$ & $0.79(0.61-1.02)$ & 0.07 & $1.75(1.27-2.40)$ & $<0.01$ \\
\hline $\mathrm{F}$ & $0.49(0.39-0.63)$ & $<0.01$ & $0.90(0.69-1.17)$ & 0.44 \\
\hline \multicolumn{5}{|l|}{ KTAS level } \\
\hline 4,5 & Reference & & Reference & \\
\hline 3 & 10.88 (7.29-16.22) & $<0.01$ & $4.74(3.15-7.13)$ & $<0.01$ \\
\hline 2 & 49.69 (33.03-74.75) & $<0.01$ & $18.17(11.93-27.66)$ & $<0.01$ \\
\hline 1 & $287.0(183.86-447.97)$ & $<0.01$ & 98.85 (61.97-157.65) & $<0.01$ \\
\hline \multicolumn{5}{|l|}{ Mode of arrival } \\
\hline Individual transportation & Reference & & Reference & \\
\hline Public ambulance service & $6.81(5.50-8.42)$ & $<0.01$ & 2.38 (1.89-2.99) & $<0.01$ \\
\hline Private ambulance service & $9.73(7.86-12.05)$ & $<0.01$ & $3.03(2.40-3.81)$ & $<0.01$ \\
\hline Age & $1.05(1.05-1.06)$ & $<0.01$ & $1.04(1.03-1.04)$ & $<0.01$ \\
\hline Patients per bed & $0.51(0.37-0.70)$ & $<0.01$ & $0.78(0.33-1.83)$ & 0.59 \\
\hline
\end{tabular}

$\mathrm{OR}$, odds ratio; $\mathrm{Cl}$, confidence interval; KTAS, Korean Triage and Acuity Scale.

strike during the COVID-19 pandemic on the mortality and ED LOS of patients who visited the EDs of six teaching hospitals in Daegu, South Korea. Doctors do not strike frequently because of ethical concerns [6]. In particular, a physicians' strike is heavily criticized because of the belief that it will adversely impact patients (e.g., delay of scheduled surgeries, failure to provide proper care for emergency patients). Contrary to popular belief, however, a previous review did not observe a clear correlation between physician strikes and mortality [1].

In the present study, the number of patients who visited the EDs of the six teaching hospitals was affected during the strike; however, this relationship varied in previous studies. Salazar et al. [7] reported that there were no marked differences in the number of patients during a strike. In a 2016 study on a junior physicians' strike, Furnivall et al. [8] observed that the number of patients who actually visited a hospital fell short of the projections. These results could be attributed to the differences in methods of measuring the impact of strikes and various sociological factors, such as whether the strike emerged as a key issue in society.

Compared to the control periods, the patients' conditions were more severe during the strike period. That is, the number of pa- tients classified as KTAS levels 1 or 2 increased during the strike period compared to that in the control periods. This result differs from that of a year 2000 study at Daejeon by Lee et al. [3], who reported an increased number of patients with mild conditions during a strike. The difference between the findings of this study and ours may be due to the low participation of primary care facility physicians in the present study's strike; thus, patients with mild conditions would have had no need to visit a tertiary hospital. Furthermore, the fact that patients with fever alone (no other symptoms) had limited access to primary care facilities during the pandemic may also have influenced the results.

COVID-19 is the first pandemic that has occurred since the Spanish flu in 1918. To the best of our knowledge, this is the first study to analyze resident strikes during such a period. We compared the impact of COVID-19 and residents' strikes on the ED. A previous study that analyzed the impact of COVID-19 on the ED reported similar outcomes [5], that is, the number of patients utilizing EDs declined. This decrease may have been due to people's fear of an increased risk of COVID-19 infection during ED visits.

We examined the impact of resident strikes on EDs in terms of mortality. In a previous study on the impact of physicians' strikes in 
public hospitals in Israel, Siegel-Itzkovich [9] analyzed the impact based on the number of funerals performed and reported that the number of funerals decreased compared to the previous 3 years without a strike. In a study regarding the effects of a physicians' strike in hospitals and polyclinics in Croatia, Erceg et al. [10] reported that there were no differences in the distributions of mortality and cause of death between the strike period and the comparison period. Ong'ayo et al. [2] analyzed the impact of strikes by healthcare providers in Kenya, including physicians, and their results showed that healthcare provider strikes were not correlated with patient mortality. Although the mortality rate in the strike period differed from that in control period 1 , it did not differ from that in control period 2 , which suggests that the difference in mortality was due to the pandemic. This is consistent with previous findings in which resident strikes did not lead to increased patient mortality. However, it should be noted that examining the impact of a strike on patients solely based on mortality is a limited approach, and additional studies are needed to develop more accurate measurement approaches.

The ED LOS decreased during the strike period compared to that in the control periods 1 and 2. This is in line with the results of a previous study on resident efficacy. Harvey et al. [11] reported that resident strikes led to a decrease in the ED LOS. Kim et al. [12] also reported that the LOS and number of tests and treatments performed in the ED declined. The decrease in ED LOS in our study may have been due to the fact that physicians avoided performing unnecessary tests and could quickly determine patient disposition. A previous study simultaneously observed a decrease in tests and procedures performed in the $\mathrm{ED}[3]$; however, our study was limited in that it did not investigate the number of tests and procedures performed in the ED.

Although we were able to determine the mortality of patients who visited the ED during a resident strike in this study, a 19-day data collection may not have accurately portrayed the potential effect. We hypothesized that a reduction in the number of medical personnel may have a negative effect on patients' treatment outcomes. However, there are some points that require further investigation in future research, such as specialists filling in for residents, additional staff deployment from other departments, and additional factors such as differences in the number of diagnostic procedures performed.

One limitation of this study was its retrospective design, as a prospective review of medical records was not a viable study option. Furthermore, it was not possible to include all patients influenced by the resident strike or those who visited outpatient clinics rather than an ED. Another limitation was that the exact strike period and control period 2 for comparing mortality were not equivalent. Al- though we tried to use the same number of days for the study and control periods, they were not set to the same time of the year, which allowed for seasonal differences. Furthermore, we did not use a clear parameter to compare the increased workload in the ED due to the COVID-19 pandemic. We used mortality and ED LOS as our study parameters for comparison, although the work intensity increased even when working for the same number of hours, for example, extra time spent donning and removing personal protective equipment, such as N95 masks and face shields. In particular, before treating patients with fever and respiratory symptoms, more time was needed to replace personal protective equipment and N95 masks between patients. COVID-19 diagnostic testing was performed only at certain times and would have affected the patients' LOS. Finally, the parameters used in our study may not reflect the pandemic situation and were based on the parameters used in previous studies. Based on the results of this study, it is necessary to perform complex analyses of ED indicators in the setting of a pandemic in future studies.

In conclusion, our findings indicated that the resident strikes did not impact patient mortality in the EDs of the six teaching hospitals in Daegu, South Korea. During the strike, the number of patients visiting the $\mathrm{ED}$ and LOS in the ED decreased.

\section{Notes}

\section{Conflicts of interest}

No potential conflict of interest relevant to this article was reported.

\section{Author contributions}

Conceptualization: YHC, HWR, JHK, TCJ, DEL; Data curation: SM, JHK, SHL, TCJ, DEL; Formal analysis: JWC, SM, JHK; Methodology, Project administration: YHC, HWR, SM, SHL; Investigation: SM, JHK, SHL, TCJ, DEL; Resources, Software: JWC, HWR, TCJ; Supervision, Validation, Visualization: HWR, SM, JHK; Writing-original draft: YHC, JWC, SM; Writing-review \& editing: YHC, JWC, HWR, SM, DEL.

\section{ORCID}

Yo Han Cho, https://orcid.org/0000-0002-8143-725X Jae Wan Cho, https://orcid.org/0000-0002-5342-155X Hyun Wook Ryoo, https://orcid.org/0000-0002-1361-9887 Sungbae Moon, https://orcid.org/0000-0001-6928-8573 Jung Ho Kim, https://orcid.org/0000-0002-3215-4640 Sang-Hun Lee, https://orcid.org/0000-0003-4303-7375 Tae Chang Jang, https://orcid.org/0000-0002-0895-5990

Dong Eun Lee, https://orcid.org/0000-0002-2057-5261 


\section{References}

1. Cunningham SA, Mitchell K, Narayan KM, Yusuf S. Doctors' strikes and mortality: a review. Soc Sci Med 2008;67:1784-8.

2. Ong’ayo G, Ooko M, Wang’ondu R, Bottomley C, Nyaguara A, Tsofa BK, et al. Effect of strikes by health workers on mortality between 2010 and 2016 in Kilifi, Kenya: a population-based cohort analysis. Lancet Glob Health 2019;7:e961-7.

3. Lee SW, Yang YM, Ha YR, Chung SP, Yoo IS, Kim SW. The impact of doctors' strike on medical care in the emergency department. J Korean Soc Emerg Med 2002;13:181-6.

4. Hartnett KP, Kite-Powell A, DeVies J, Coletta MA, Boehmer TK, Adjemian J, et al. Impact of the COVID-19 pandemic on emergency department visits: United States, January 1, 2019May 30, 2020. Morb Mortal Wkly Rep 2020;69:699-704.

5. Wong LE, Hawkins JE, Langness S, Murrell KL, Iris P, Sammann A. Where are all the patients? Addressing Covid-19 fear to encourage sick patients to seek emergency care. NEJM Catal Innov Care Deliv 2020;May 14 [Epub]. https://doi.org/10. 1056/CAT.20.0193.

6. Chima SC. Doctor and healthcare workers strike: are they ethi- cal or morally justifiable: another view. Curr Opin Anaesthesiol 2020;33:203-10.

7. Salazar A, Corbella X, Onaga H, Ramon R, Pallares R, Escarrabill J. Impact of a resident strike on emergency department quality indicators at an urban teaching hospital. Acad Emerg Med 2001;8:804-8.

8. Furnivall D, Bottle A, Aylin P. Retrospective analysis of the national impact of industrial action by English junior doctors in 2016. BMJ Open 2018;8:e019319.

9. Siegel-Itzkovich J. Doctors' strike in Israel may be good for health. BMJ 2000;320:1561.

10. Erceg M, Kujundzić-Tiljak M, Babić-Erceg A, Coric T, Lang S. Physicians' strike and general mortality: Croatia's experience of 2003. Coll Antropol 2007;31:891-5.

11. Harvey M, Al Shaar M, Cave G, Wallace M, Brydon P. Correlation of physician seniority with increased emergency department efficiency during a resident doctors' strike. $\mathrm{N} Z \mathrm{Med} J$ 2008;121:59-68.

12. Kim SG, ChungJY, Jeong JW, Cho SJ. Effectiveness of emergency management by the medical staff in the emergency department. J Korean Soc Emerg Med 2003;14:500-7. 